\title{
IR contamination in quiescence X-Ray Novae
}

\author{
Mark T. Reynolds, Paul J. Callanan and Colm Kelleher \\ Department of Physics, University College Cork, Ireland \\ email: m.reynolds@ucc.ie
}

\begin{abstract}
We present archival optical-IR observations of a number of short period X-ray novae (XRNe). We use these to show that in contrast to the current paradigm, there is likely significant non stellar flux in the infra-red (IR).
\end{abstract}

Keywords. Stars: individual (XTE J1118+480, GRO J0422+32, A0620-00, GS2000+25, GS1142-68 \& Cen X-4) - X-rays: binaries

\section{Introduction}

Observations of X-ray novae (XRNe) in quiescence are an important means by which it has been possible to constrain the mass of the compact object in accreting binaries (see Charles \& Coe (2006) for a thorough review). Such observations are easiest to perform in the optical; however, contamination from the accretion disc can be significant here, particularly for measurements of the ellipsoidal modulation. As such observations in the IR are to be preferred, where the flux from the secondary star is thought to be dominant (Charles \& Coe 2006).

Previous quiescent IR observation of the XRNe V404 Cyg and A0620-00 (Shahbaz et al. 1996, 1999) have been used to limit the IR contamination from the accretion disc to be $\leqslant 14 \%$ and $27 \%$ respectively. However, recent K-band observations of GRO J0422+32 by Reynolds et al. (2006) appear to show a light curve dominated by emission from the accretion disc and not the ellipsoidal modulation of the secondary star, see Fig. 1. Here, we take a photometric approach to constraining any accretion disk contribution to the IR flux of quiescent XRNe: we use the measured secondary star contribution in the optical to predict its contribution to the overall flux at longer wavelengths. We will show that there appears to be a significant excess of flux, above that expected from the secondary alone, present in the IR.

The data consist of the optical-IR (including Spitzer data where possible) spectral energy distribution (SED) of a number of XRNe consisting of data taken from the literature, namely : XTE J1118+480, GRO J0422+32, A0620-00, GS2000+25, GS1142-68 \& Cen X-4.

\section{Spectral Energy Distributions}

In contrast to previous authors who normalised to the K-band flux (Muno et al. 2006; Gelino, et al. 2003; Gelino et al. 2001), where the IR contamination from the accretion disc is often ill-unconstrained, we choose to normalise the model atmospheres of the appropriate spectral type to the measured secondary contribution in the R-band (which has been directly measured for these systems). We fit a NextGen model atmosphere (Hauschildt et al. 1999a; Hauschildt et al. 1999b) corresponding to the spectral type of the secondary star in each system. 


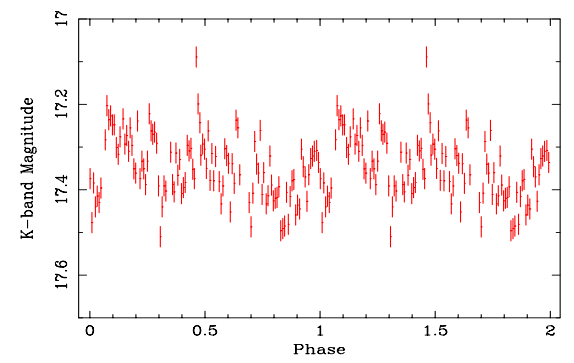

Figure 1. 2 minute resolution K-band light curve of GRO J0422+32. Two orbital phases are displayed for added clarity.

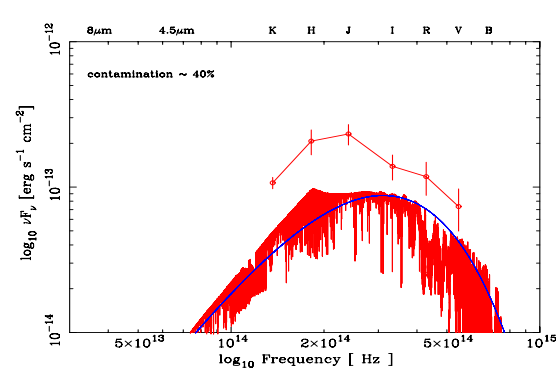

Figure 2. The optical-IR SED of GRO J0422+32.

\section{Discussion}

It is immediately apparent, when normalising to the flux in this way, that there is significant non-stellar flux in the IR, see Fig. 2. We note that there is a systematic excess of flux in the IR in all 6 systems. In each system the excess flux at IR wavelengths is similar to that observed in the optical. It is unclear why such systematic differences should occur for all 6 systems. However, we cannot rule out the following possibilities;

- There is a systematic under-estimation of the extinction towards the XRNe.

- The optical veiling is systematically over estimated.

The data suggest an alternative explanation: that the IR flux for these systems may have a significant component that does not originate from the secondary. The spectral shape of the IR excess we observe is consistent with having a blackbody origin. Hence, the observed excess is unlikely to be synchrotron in nature and is instead more likely to originate in a circumbinary disc or the cool outer regions of the accretion disc.

\section{Conclusions}

The current paradigm assumes that the observed IR flux from XRNe is dominated by emission from the secondary; however, the analysis presented here may cast some doubt on this assumption. If confirmed, the existence of a significant IR excess in these systems may have important consequences for any mass estimates of the compact object that use IR ellipsoidal modulation measurements.

\section{Acknowledgements}

This research made use of the SIMBAD database, operated at CDS, Strasbourg, France, and NASA's Astrophysics Data System. The authors wish to acknowledge financial support from Science Foundation Ireland.

\section{References}

Charles, P. A. \& Coe, M. J., 2006, in Lewin W.H. G., van der Klis M., eds, Compact Stellar $\mathrm{X}$-Ray Sources (Cambridge University Press, Cambridge)

Gelino, D. M., Harrison, T. E. \& Orosz, J. A., 2001, AJ, 122, 2668

Gelino D. M. \& Harrison T. E., 2003, ApJ, 599, 1254

Hauschildt, P. H., Allard, F. \& Baron, E., 1999, ApJ, 512, 377

Hauschildt, P. H., Allard, F., Ferguson, J., Baron, E. \& Alexander, D. R., 1999, ApJ, 525, 871

Muno, M. P. \& Mauerhan, J., 2006, ApJ, 648, 135

Reynolds, M. T., Callanan, P. J. \& Filippenko, A. V., 2006, MNRAS submitted (astro$\mathrm{ph} / 0610272)$

Shahbaz, T., Bandyopadhyay, R., Charles, P. A. \& Naylor, T., 1996, MNRAS, 282, 977

Shahbaz, T., Bandyopadhyay, R. \& Charles, P. A., 1999, A\&A, 346, 82 